\title{
Clinical Analysis of Dispelling Wind, Eliminating Lung and Relieving Cough Combined with Western Medicine in the Treatment of Cough Variant Asthma in Children
}

\author{
He Huang* \\ Nanjing Hospital of Traditional Chinese Medicine Affiliated to Nanjing University of Chinese medicine (Nanjing Hospital \\ of traditional Chinese Medicine), Nanjing 210018, Jiangsu Province, China \\ *Corresponding author: He Huang, 2388411202@qq.com

\begin{abstract}
Objective: This study mainly explores the clinical effect of dispelling wind, eliminating lung and relieving cough combined with western medicine in the treatment of cough variant asthma. Methods: 80 children with cough variant asthma accepted by our hospital from January 2018 to December 2020 were randomly selected for the study and divided into two groups. One group was the reference group (40 cases) treated with procaterol hydrochloride tablets and montelukast sodium, and the other group was the research group (40 cases). The method of eliminating wind, eliminating lung and relieving cough was combined with procaterol hydrochloride tablets and montelukast sodium to observe and compare the curative effects of the two groups. Results: There was no significant difference in TCM symptom score and eosinophil (EOS) count between the two groups before treatment $(\mathrm{P}>0.05)$; After treatment, the TCM symptom scores of coughs, pharyngeal itching, expectoration, nasal congestion and nasal itching in the research group were lower than those in the reference group, and the EOS count was lower than that in the reference group $(\mathrm{P}<0.05)$; The effective rate of research group was higher than that of reference group $(\mathrm{P}<0.05)$. Conclusions: For children with cough variant asthma, Qufeng Sufei cough relieving method combined with procaterol hydrochloride and montelukast sodium can improve children's symptoms and reduce eosinophil count.
\end{abstract}

Keywords: Dispelling wind; Eliminating lung and relieving cough; Procaterol hydrochloride; Montelukast sodium; Children; Cough variant asthma; Clinical effect

Publication date: September 2021; Online publication: September 30, 2021

\section{Introduction}

Cough variant asthma CVA is a special type of bronchial asthma in children and the main cause of chronic cough in children. When children suffer from cough variant asthma, the main clinical manifestations are repeated cough and dry cough. It often occurs at night or early in the morning. It is characterized by cough attack or aggravation when smelling peculiar smell, intense activity, crying, dietary stimulation, etc, If the child's condition persists, it may even develop into typical asthma, which will seriously affect the child's quality of daily life and growth ${ }^{[1]}$. In addition, in recent years, the changes of urban living environment and pace of life, related urban pollution and academic pressure have also led to the gradual increase of the incidence of cough variant asthma. Once it is ill, it needs to be treated as soon as possible. At present, drugs are often used in clinical treatment, such as bronchodilators, glucocorticoids and leukotriene receptor antagonists. Although they can improve the symptoms of children to a certain extent, the drugs have certain side effects, and the disease may relapse after stopping the drugs, so the limitations of drugs are high, 
Therefore, it is of great clinical value to explore a more reasonable and safe treatment scheme ${ }^{[1]}$. The syndrome of wind phlegm blocking lung in children with cough variant asthma is more common. The method of eliminating wind, eliminating lung and relieving cough combined with western drugs procaterol hydrochloride tablets and montelukast sodium can quickly improve children's symptoms and reduce eosinophil count.

\subsection{Basic data}

The subjects of this study are 80 children with cough variant asthma treated in our hospital from January 2018 to December 2020. The children are divided into two groups by random number table method. One group is the reference group, 40 cases, the proportion of men and women is $23: 17$, the age is between 3-12 years old, the average age is $(6.71 \pm 1.05)$ years old, the shortest course of disease is 2 months, the longest is 6 months, and the average course of disease is $(2.78 \pm 0.24)$ Months; The first group was research group, 40 cases, with a male to female ratio of $24: 16$, aged between $3-11$ years, with an average age of $(6.68 \pm$ 1.01) years. The shortest course of disease was 2 months and the longest was 5 months, with an average course of disease of $(2.94 \pm 0.25)$ months; There was no significant difference in the above data $(\mathrm{P}>0.05)$.

\subsubsection{Inclusion criteria:}

Diagnostic criteria of Western medicine: It meets the diagnostic criteria of CVA in the guidelines for the diagnosis and treatment of chronic cough in Chinese children (revised in 2013) ${ }^{[2]}$ :

(1) Cough for more than 1 month, manifested as dry cough, no wheezing, more than vigorous exercise, attack or aggravation at night and early morning

(2) The individual or first and second degree relatives have a history of allergic diseases and the allergen test is positive

(3) Antibiotic treatment was ineffective and there was no infection

(4) The use of anti-asthma drugs is effective

(5) The diurnal variability of bronchial provocation test or peak expiratory flow was positive.

\subsubsection{TCM Syndrome Differentiation Standard}

Refer to the clinical diagnosis and treatment guidelines of Pediatrics of traditional Chinese medicine children's cough variant asthma ${ }^{[3]}$ revision of wind phlegm blocking lung syndrome.

\subsubsection{Symptoms see}

Dry cough, sticky phlegm, difficult cough, itchy nose, stuffy nose, sneezing, itchy throat, red tongue, white and greasy fur, slippery pulse.

\subsubsection{Inclusion criteria}

(1) Meet the above diagnostic criteria of Western medicine and TCM syndrome differentiation criteria

(2) Children in clinical attack stage

(3) All patients in this study were informed and signed the study consent.

\subsubsection{Exclusion criteria}

(1) Children with chronic cough caused by chronic bronchitis, pertussis, cardiogenic cough and other factors 
(2) Dysfunction of liver, kidney, heart and lung and other important organs

(3) Poor treatment compliance and incomplete clinical data affect the efficacy evaluation

(4) Allergic to therapeutic drugs.

\section{Method}

Reference group: Procaterol hydrochloride tablets $(25 \mu \mathrm{g})$ (gyzz h10930017 of Zhejiang Otsuka Pharmaceutical Co., Ltd.), half a tablet for 3-5 + years old, 1 tablet for 6-12 years old, twice a day, in the morning and before going to bed; Montelukast sodium chewable tablets (Hangzhou moshadong Pharmaceutical Co., Ltd. 5mg / tablet, gyzz j20130054 4mg / tablet, gyzz j20130053), 3-5 + years old 4mg / time, once a day, 6-12 years old 5mg / time, once a day, lasting for 14 days.

On the basis of the above, research group developed Qufeng Sufei Zhike Decoction by the author. The basic formula is as follows: $10 \mathrm{~g}$ mulberry leaves, $10 \mathrm{~g}$ mulberry peel, $9 \mathrm{~g}$ almond, $6 \mathrm{G}$ Suzi, $10 \mathrm{~g}$ Peucedanum, $10 \mathrm{~g}$ honey roasted loquat leaves, 6G dry earthworm, 6G cicada molting, 6G Magnolia (fried), 10g fried beef seeds, 6G tangerine peel and 5g licorice;3-4 years old, light weight, dose reduction; Add or subtract with symptoms. If cough occurs frequently, loquat leaves can be added to $15 \mathrm{~g}$, runny nose is clear and thin, and Schizonepeta tenuifolia and Fangfeng can be added; Pharyngeal itching, obvious pharyngeal pain, plus mint and forsythia; Yellow phlegm, red tongue and yellow fur, plus Scutellaria and gardenia; Decoct 150$200 \mathrm{ml}$ of soup with water, take it twice in the morning and evening or a small amount frequently, and continue the treatment for 14 days. If the symptoms are obviously relieved in the second week, the medium dose of the prescription shall be reduced.

\subsection{Observation indicators}

The TCM symptom scores of the two groups before and after treatment were observed and compared. The symptoms were cough, pharyngeal itching, expectoration, nasal congestion and nasal itching. The scores were $0,1,2,3$ and 4 respectively. The corresponding symptom degrees were asymptomatic, mild, moderate and severe symptoms. The lower the score, the higher the effect of symptom improvement. The changes of eosinophil count (EOS) were detected before and after treatment.

Clinical effect evaluation: After treatment, the symptom score decreased by more than $70 \%$, and the curative effect was significant; If the symptom score of children is reduced by $50-70 \%$, the effect evaluation is effective; If the symptom score is not reduced after treatment, the effect evaluation is invalid; Total effective rate $=($ markedly effective + effective $) /$ total number of cases $\times 100 \%{ }^{[4]}$.

\subsection{Statistical analysis}

SPSS 23.0 processing and analysis, using $T$ and $X^{2}$ for test, and expressed in $(\bar{x} \pm s)(n / \%)$. If $P<0.05$, there is a difference in the data.

\section{Results}

\subsection{Observe the improvement of symptoms}

Table 1. shows the statistical data of symptom scores of the two groups. There was no significant difference between the two groups before treatment, $\mathrm{P}>0.05$; After treatment, the symptom scores of coughs, pharyngeal itching and expectoration in the research group were lower than those in the reference group (P $<0.05)$.

\subsection{Statistics and comparison of eosinophil count changes}

There was no significant difference in eosinophil count between the two groups before treatment $(\mathrm{P}>0.05)$; 
the eosinophil count of the two groups decreased after treatment, and the research group was lower than that of the reference group $(\mathrm{P}<0.05)$ (Table 2).

\subsection{Observe and compare the effective rate of treatment}

The curative effect evaluation statistics showed that the effective rate of research group was higher than that of reference group $(\mathrm{P}<0.05)$ (Table 3).

Table 1. Comparison of TCM symptom scores before and after treatment $(\bar{x} \pm s)$

\begin{tabular}{cccccc}
\hline Time & Group & Cough & $\begin{array}{c}\text { Pharyngeal } \\
\text { itching }\end{array}$ & Expectoration & $\begin{array}{c}\text { Nasal congestion } \\
\text { and itching }\end{array}$ \\
\hline Before & Reference group & $2.51 \pm 0.34$ & $2.42 \pm 0.25$ & $1.01 \pm 0.25$ & $2.35 \pm 0.41$ \\
treatment & Research group & $2.53 \pm 0.29$ & $2.39 \pm 0.28$ & $1.02 \pm 0.34$ & $2.41 \pm 0.42$ \\
t & & 0.2831 & 0.5055 & 0.1499 & 0.6465 \\
P & & 0.7779 & 0.6147 & 0.8813 & 0.5198 \\
After & Reference group & $1.87 \pm 0.28$ & $1.84 \pm 0.22$ & $0.61 \pm 0.17$ & $1.05 \pm 0.25$ \\
treatment & Research group & $1.45 \pm 0.21$ & $1.21 \pm 0.15$ & $0.42 \pm 0.15$ & $0.58 \pm 0.14$ \\
t & & 7.5895 & 14.9640 & 5.3003 & 10.3742 \\
P & & 0.0000 & 0.0000 & 0.0000 & 0.0000 \\
\hline
\end{tabular}

Table 2. Comparison of eosinophil count $\left(\bar{x} \pm \mathrm{s}, 10^{9} / \mathrm{L}\right)$ between the two groups before and after treatment

\begin{tabular}{cccc}
\hline Group & N (example) & Before treatment & After treatment \\
\hline Reference group & 40 & $0.84 \pm 0.23$ & $0.61 \pm 0.11$ \\
Research group & 40 & $0.82 \pm 0.21$ & $0.41 \pm 0.09$ \\
t & & 0.4308 & 9.4398 \\
P & & 0.6677 & 0.0000 \\
\hline
\end{tabular}

Table 3. Comparison of effective rates between the two groups

\begin{tabular}{cccccc}
\hline Group & N (example) & $\begin{array}{c}\text { Remarkable } \\
\text { effect }\end{array}$ & Effective & invalid & $\begin{array}{c}\text { Total effective } \\
\text { rate }\end{array}$ \\
\hline $\begin{array}{c}\text { Reference } \\
\text { group }\end{array}$ & 40 & 14 & 16 & 10 & 75.00 \\
Research group & 40 & 17 & 20 & 3 & 92.50 \\
$X^{2}$ & & & & & 4.5006 \\
P & & & & & 0.0338 \\
\hline
\end{tabular}

\section{Discussion}

As a special type of asthma, cough variant asthma has repeated attacks, which will have a great impact on children's growth and development and quality of life. It must be treated as soon as possible. In the current routine treatment of Western medicine, procaterol hydrochloride tablets can inhibit acetylcholine, block cough reflex, have anti-allergy, and improve patients' pulmonary edema and respiratory hyperresponsiveness. As a leukotriene receptor antagonist, montelukast sodium can bind to leukotriene receptor, effectively inhibit inflammatory factors, and block the maturation process of eosinophils, so as to improve children's symptoms, reduce airway inflammatory infiltration and alleviate airway 
hyperresponsiveness ${ }^{[5]}$.

From the analysis of traditional Chinese medicine, the occurrence of cough variant asthma is mainly related to the function of eliminating phlegm, wind and depression in the lung. The pathogenesis is mainly wind phlegm blocking the lung and loss of depression in the lung. Therefore, the main principle of treatment is to dispel weathered phlegm and eliminate lung and reduce Qi. Using the author's self-made Qufeng Sufei Zhike decoction can give play to its unique advantages. In the decoction, mulberry leaves can clear the lung and moisten dryness, mulberry white skin can relieve lung and asthma, almond can dispel phlegm and relieve cough, and perilla has the effects of moistening intestines, relieving defecation, relieving fever and relieving cough; Qianhu has the functions of reducing Qi and resolving phlegm, dispersing wind and clearing heat. Loquat leaves have the effects of clearing lung and stomach, reducing Qi and resolving phlegm; Dry earthworm has the effect of clearing lung and relieving asthma; Cicada molting has the functions of evacuating wind heat and relieving cough. Magnolia can dispel wind cold, dredge nose orifices, stir fry cow seeds to facilitate swallowing, orange peel to regulate qi, produce licorice, clear heat and detoxify, and roasted licorice can reconcile various drugs.

In addition, according to modern pharmacological research, mulberry leaves contain flavonoids, coumarins and steroids, which can play an anti-inflammatory role. The bitter almond contained in almond can inhibit the respiratory center to a certain extent, so as to achieve the effect of relieving cough and asthma, and improve immune function ${ }^{[6]}$; Perilla seed contains Perillaldehyde, which can dilate skin blood vessels, reduce bronchial secretion, reduce bronchospasm, and have the effect of relieving cough and expectoration; Loquat leaves contain amygdalin, which can play the role of expectorant and anti-asthmatic; Loquat leaves contain loquat glycosides and ursolic acid, which have antitussive effects, and can play anti-inflammatory and anti-allergic effects; Gandilong has sedative and anticonvulsant effects, and the Lumbrokinase component can improve hemorheology, inhibit platelet aggregation, block histamine receptor and play an anti-asthmatic role to a certain extent; Magnolia contains volatile oil and other components, which has the effects of anti-inflammatory, analgesic and improving microcirculation; The components contained in licorice can produce glycyrrhetinic acid, which can reasonably inhibit inflammation and have anti allergic effect ${ }^{[7]}$. Clinical practice research found that on the basis of routine western medicine treatment programs of procaterol hydrochloride and montelukast sodium, combined with Qufeng Sufei Zhike formula, it can play a synergistic role, give full play to their respective treatment advantages, enhance clinical efficacy, help children quickly control disease symptoms and improve their quality of life. In this study, the TCM symptom scores of the research group were lower than those of the reference group, and the eosinophil count index was lower than that of the reference group. The treatment efficiency was higher than that of the reference group, and the data comparison had statistical difference.

In conclusion, Qufeng Sufei antitussive method combined with western medicine can play a significant effect on cough variant asthma, reduce eosinophil count and improve clinical symptoms, which can be popularized and applied in clinic.

\section{Disclosure statement}

The author declares no conflict of interest.

\section{References}

[1] Wang S, Chen L, 2019, Effects of budesonide inhalation combined with montelukast sodium on serum IL-6 and TNF in children with cough variant asthma- $\alpha$, TGF- $\beta$ And IgE level and its mechanism. Journal of Modern Laboratory Medicine, 34(01): 143-147. 
[2] Chronic cough collaborative group, respiratory group, scientific branch, Chinese Medical Association, Editorial Committee of Chinese Journal of Pediatrics, 2014, Guidelines for the diagnosis and treatment of chronic cough in Chinese children (revised in 2013). Chinese Journal of Pediatrics, 52(3): 184-188.

[3] Feng X, Duan X, Sun L, et al., 2016, Guidelines for clinical diagnosis and treatment of Pediatrics of traditional Chinese medicine. Pediatric cough variant asthma (formulated). Journal of Pediatrics of Traditional Chinese Medicine, 12(5): 1-4.

[4] Li J, Sun J, Li Z, 2020, Efficacy of montelukast sodium combined with procaterol in the treatment of chronic cough and its effects on pulmonary function, serum IL-4 and TNF in children- $\alpha$, Effects of Ig E and EOS levels. Hainan Medical Science, 031(011): 1401-1404.

[5] Tang W, Xu H, Xu J, et al., 2017, Effects of montelukast sodium combined with budesonide suspension on respiratory function, blood IgE and EOS in children with cough variant asthma. Chinese Journal of Medical Frontier (Electronic Edition), 9(12): 134-138.

[6] He S, Li L, 2018, Clinical study on Xiaoke Pingchuan decoction combined with terbutaline and montelukast sodium in the treatment of cough variant asthma in children. Modern Medicine and Clinic, 33(010): 2709-2712.

[7] $\mathrm{Hu} \mathrm{N}, \mathrm{Xu} \mathrm{X}$, Tang L, 2019, Efficacy of montelukast sodium combined with Cetirizine Hydrochloride in the treatment of cough variant asthma in children and its effect on serum NT-3 and EOS levels. Journal of Clinical Lung, 24(12): 2207-2211. 